\title{
Transmission of KPC producing Klebsiella pneumoniae despite appropriate barrier precautions of an intensive care unit in the Netherlands
}

\author{
B Diederen ${ }^{1 *}$, C Hattink $^{2}, M$ de Groot ${ }^{3}$ \\ From International Conference on Prevention \& Infection Control (ICPIC 2011) \\ Geneva, Switzerland. 29 June - 2 July 2011
}

\section{Introduction / objectives}

Enterobacteriaceae producing carbapenemases are very rare in the Netherlands and correspond almost exclusively to imported clones from endemic areas. Here, we report an imported case of KPC-carrying $K$. pneumoniae with transmission to another patient.

\section{Methods}

A 68 year old female who was travelling in Greece was admitted to the ICU of the University Hospital of Ioannina in Greece for urosepsis and hypercapnic coma. The patient was transferred to the ICU of the Spaarne Hospital on September $22^{\text {nd }} 2010$ (day 9). Patient was admitted in strict barrier precautions for MRSA. Intestinal carriage of MDR Enterobacteriaceae was screened at admission and a carbapenem-resistant $K$. pneumoniae strain was isolated from a throat swab. A confirmational PCR was positive for blaKPC. On October 14th a routine urine sample from a patient who had been admitted for 15 days at the ICU, tested positive for a genotypically identical KPC producing K. pneumoniae.

\section{Results}

Secondary spread was investigated by active surveillance. None of the patients or personnel was found to be positive for KPC-carrying Enterobacteriaceae. No further cases were identified.

\section{Conclusion}

Here we report dissemination of KPC-producing $K$. pneumoniae from a patient repatriated from a Greece ICU despite continuous barrier precautions. We found no evidence for further local spread within our hospital. We stress the importance of early identification and confirmation followed by intensified infection control measures to prevent the dissemination of Enterobacteriaceae with KPC-enzymes.

\section{Disclosure of interest}

None declared.

\section{Author details}

${ }^{1}$ Regional Laboratory of Public Health Kennemerland, Haarlem, Netherlands. 2Department of Infection Control, Spaarne Ziekenhuis, Hoofddorp,

Netherlands. ${ }^{3} \mathrm{ICU}$ Department, Spaarne Ziekenhuis, Hoofddorp, Netherlands.

Published: 29 June 2011

doi:10.1186/1753-6561-5-S6-O25

Cite this article as: Diederen et al:: Transmission of KPC producing Klebsiella pneumoniae despite appropriate barrier precautions of an intensive care unit in the Netherlands. BMC Proceedings 2011 5(Suppl 6): 025. 